\section{PERFORATIVE DIVERTICULITIS OF THE COLON}

\author{
BY
}

\author{
STANLEY N. SENNETT, M.B., B.S.LOND., \\ M.D. BERL. \\ CAPETOWN, SOCTH AFrica \\ (With Special Plate)
}

Of the conditions which may give rise to an acute abdomen, a perforative diverticulitis of the colon is comparatively rare, and it is still rarer for the diagnosis to be made with any degree of confidence. Such a case diagnosed correctly on clinical grounds was described by Sir I. de C. Wheeler in the British Medical Journal of January 4th, 1930.

Recently it was possible for me to recognize a case of perforative diverticulitis with tolerable certainty, as a few days after I had diagnosed diverticulitis with the aid of a barium enema, the patient developed an acute abdomen.

The patient, a Norwegian air pilot, aged 40, came to see me on December 29th, 1930. He kad had some pain in the lower abdomen for ten days previously. For the first eight days the pain was not bad, but for the last two days it was very severe and kept him awake all night. It came in spasms, some of them being so severe as to double him up and make him shout. The pain was not related to food, yet he was afraid to eat lest it came on again. His appetite was very good, and he felt quite comfortable in the intervals between the attacks. He had had similar pains about one year previously in Norway, but was put on a " diet," and had then no further trouble.

During the attacks he found it painful to urinate. IIe had frequency of micturition by day, and at night waked once or twice. He was also somewhat constipated. His tongue was furred, and there was definite tenderness in the lower abdomen, in both iliac fossae, and in the middle line. The liver and spleen were not palpable. He had large external haemorrhoids. The urine was normal.

He was ordered an enema, which made him more comfortable. The following day he was given a barium enema, which showed that the pelvic colon was markedly pulled over to the right iliac fossa, and a number of diverticula were seen. $\mathrm{He}$ was then given a barium meal ; after twenty-four hours, examination again revealed the presence of multiple diverticula and the sigmoid flexure pulled over to the right. I therefore came to the diagnosis of diverticulitis with adhesions of the sigmoid colon to structures in the right iliac fossa. This was on December 31st, 1930. His pains had greatly diminished, and he was feeling quite well.

At 8 a.m. on January 2nd, 1931, his pains came on again, but now with much greater severity. He vomited twice, and I was called to see him at $2 \mathrm{p} . \mathrm{m}$. He had a temperature of $101^{\circ}$, and his pulse was 100 . There was great tenderness and marked rigidity of the lower abdomen, particularly on the right side. He looked ill, and there was little doubt that he had an acute abdomen. Armed with the $x$ rays, I diagnosed a perforative diverticulitis. Mr. Lindsay Sandes was immediately called in. He agreed with the diagnosis, and operated the same evening. On entering the abdomen a large quantity of pus was immediately seen. A thickened, boggy loop of pelvic colon was found in the right iliac fossa, with a perforation of a diverticulum. A number of other diverticula of the sigmoid were also readily seen. The perforation was carefully closed, the pelvic colon brought back to the left iliac fossa, and the abdomen drained. The patient made a complete recovery.

He was $x$-rayed again (barium enema) on February 13th, 1931, when the loop of the pelvic colon was then seen in the left iliac fossa. Numerous diverticula were still visible in the pelvic colon. The next barium enema flowed well, showing no obstruction at the site of perforation, although the loop of the colon seemed bound down with adhesions.
COMMENTARY

Certain points of interest emerge from a consideration of the above case :

1. The fact that a barium enema examination was performed and a diverticulitis diagnosed a few days before its perforation.

2. The patient was quite well for three days after the barium enema examination, so that it cannot be held responsible for influencing the perforation.

3. Perforation of the sigmoid colon is generally fraught with grave danger, as the contents are notoriously septic. Immediate operation in the above case was followed by a complete recovery.

I wish to record my thanks to Mr. Lindsay Sandes for the manner in which he performed an admirable operation with great success.

\section{A CALCULUS IN AN ECTOPIC KIDNEY}

BY

\section{J. LLEWELLYN DAVIES, M.B., F.R.C.S.}

HONORARY ASSISTANT SURGEON IN CHARGE OF CASUALTY DEPARTMTENT, NOTTINGHAM GENEKAL HOSPITAL

(With Special Plate)

A woman, aged 34 , was admitted, in July, 1929, to the Nottingham General Hospital, under the care of Mr. F. C. Greig, complaining of abdominal pain and increased frequency of micturition, which had persisted during the past three years. The pain was referred to the lumbar region, to the left iliac fossa, and at times was said to shoot down the inner side of the right thigh. It was usually aching in character, was made worse by exercise, but was relieved by rest. Increased frequency of micturition had recently become very marked, amounting to twelve times during the day and three times at night. For the past eighteen months the act had been painless. About three years ago, for three weeks, haematuria was severe, but since then no blood has been noticed. Seven years ago hysterectomy was done, but no details are available.

On examination the woman appeared to be in fairly good health. There was some tenderness deep to the upper half of the median suprapubic operation scar. The right kidney could not be felt ; the lower pole of the left was palpable. The urine was alkaline, and contained pus. An $x$-ray photograph of the pelvis is reproduced in Fig. 1 (on Plate), and shows an unusual object situated in front of the sacrum.

At Mr. Greig's request I carried out a urological investigation. The cystogram reproduced in Fig. 2 proves that the object seen in Fig. 1 is situated above and distinct from the bladder. Cystoscopy revealed a normal bladder and left ureteric orifice, but the right ureteric orifice was red and oedematous, emitting only an occasional sluggish purulent efflux. After intravenous indigo-carmine, there were copious well-coloured effluxes from the left side in six minutes, but no dye appeared on the right side after fifteen minutes. A catheter passed up the left ureter easily, but could be introduced only a few centimetres on the right side. The pyelogram of the left kidney is normal. On the right side the catheter has passed inwards towards the promontory of the sacrum, and in one film a little of the pyelographic medium can be seen to have passed down on to the object in the pelvis.

The diagnosis suggested was a dendritic calculus in an ectopic kidney, and nephrectomy was advised.

Mr. Greig opened the abdomen through a suprapubic paramedian incision, and found the kidney -fixed retroperitoneally in the hollow of the sacrum, with a coil of ileum partly covering it and firmly adherent. The pelvis of the kidney was lying anteriorly with the ureter coming off it and passing down to the right side of the bladder. The renal artery was defined with difficulty owing to the perinephritis, and whether it arose from the right common 


\section{STANLEY N. SENNETT: PERFORATIVE DIVERTICULITIS OF THE COLON}
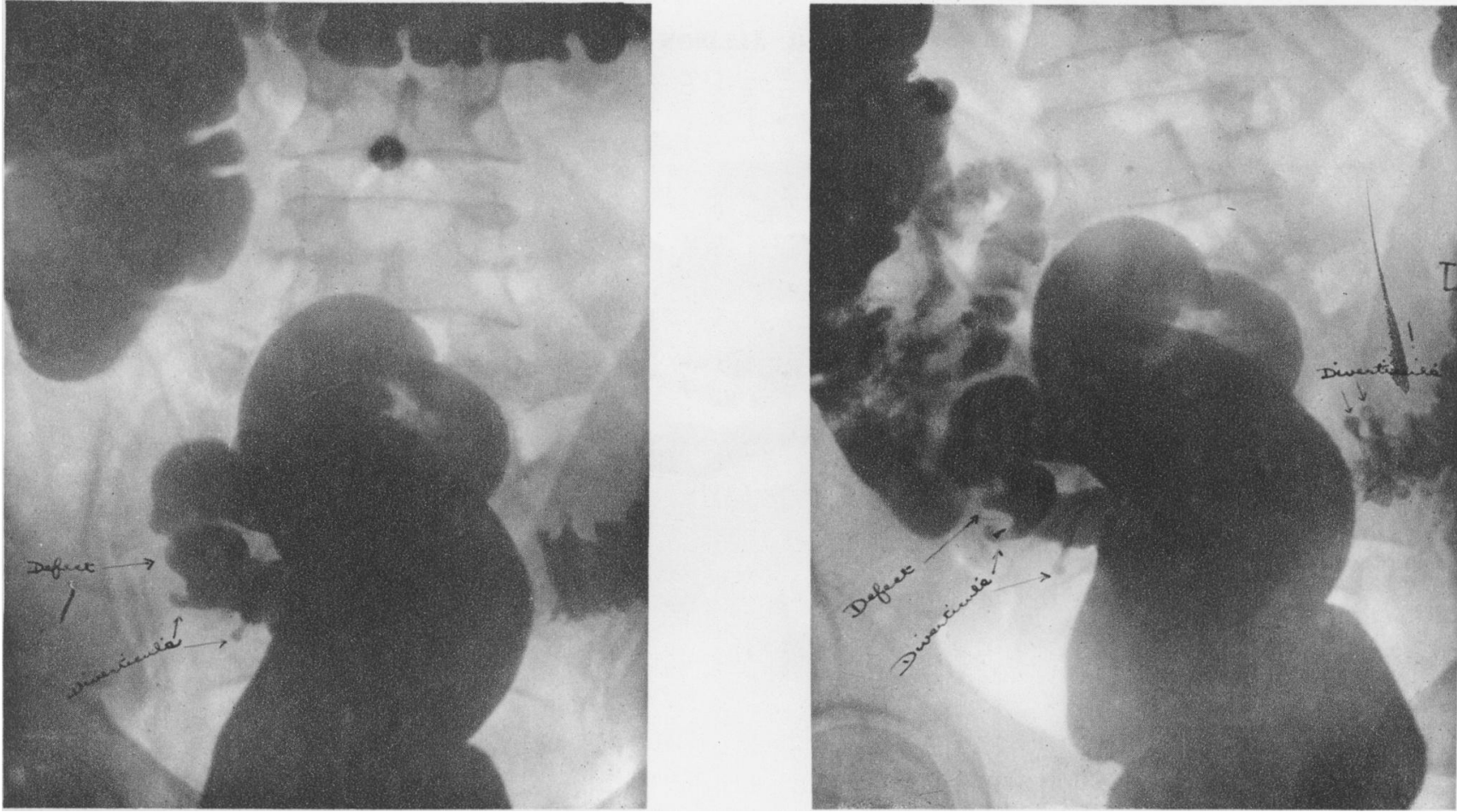

Fias. 1 Asp 2.-Two films of the barium enema examination of December 30, 1930

fossa. In Fig. 2, the loop is seen in close proximity to colls of the llellm. Two long diverticulap of the sigmoid colon is shown drawn over to the right lliac diverticula on the left. There is a "defect" shown in the loop on the right. This part of the bowel was very much thickened at operation and the perforation occurred from the diverticulum at that site.

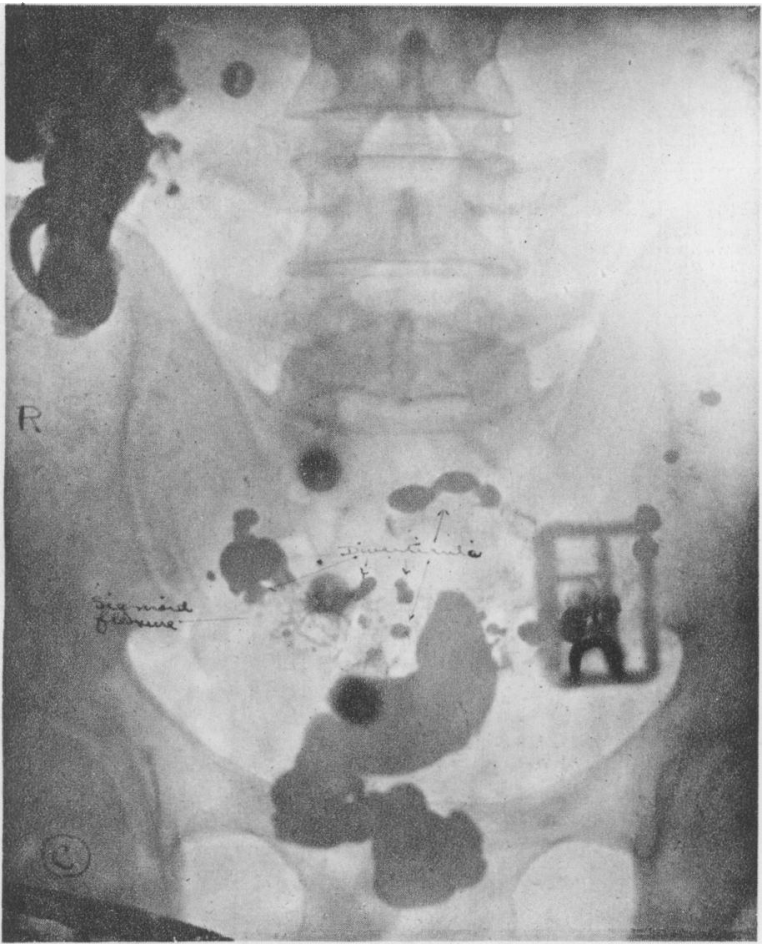

Fig. 3.-Twenty-four hours' barium meal. The outline of the sigmoid can be made out; it is drawn to the right. Numerous diverticula are shown.

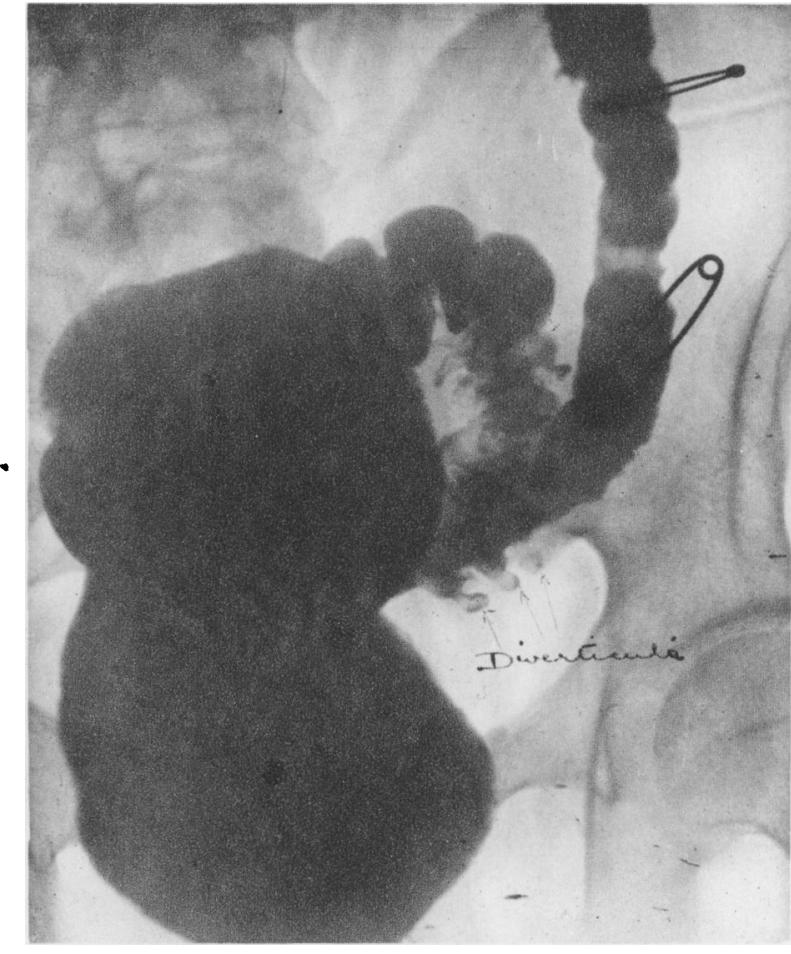
Fig. 4.-Barium enema of February 13, 1931. The sigmoid flexure
is now shown well over to the left. Beveral long diverticula can easily he seen. 